\title{
Enfermedad periodontal en pacientes con discapacidad en custodia versus pacientes con discapacidad independientes
}

\section{Resumen}

Objetivo: Identificar el estado de salud periodontal en pacientes con discapacidad en custodia versus pacientes independientes en su higiene oral. Material y métodos: Se examinaron pacientes con discapacidad, ambos sexos, 3 a 19 años que acuden al Servicio de Odontopediatría del Centro de Rehabilitación Infantil Teletón (CRIT) Tamaulipas mediante revisión de cavidad oral y aplicación del Índice de Higiene Oral Simplificado (IHOS), Índice Periodontal Comunitario de la Necesidad de Tratamiento (IPCNT) y entrevista con su cuidador primario. Se agruparon en dos categorías: Independientes y de Custodia. Resultados: En el total de la muestra la Media y Desviación estándar (D.E.) de IPCNT fue de $0.89 \pm 0.54$, así como el IHOS de $1.88 \pm 0.77$. El IPCNT en pacientes con discapacidad de Custodia fue $0.91 \pm 0.57$ e Inde-
Fermín-Guerrero-Del Ángel ${ }^{1}$

Lilia Alejandra-Dávila-Salazar ${ }^{2}$

Hilda-Issasi-Hernández ${ }^{3}$

Enrique E-Huitzil -Muñoz

Ariel-Cabrera-Salazar ${ }^{5}$

Marcela-Fragoso-Benítez ${ }^{6}$

Rogelio-Oliver-Parra ${ }^{7}$

José Martín-Torres-Benitez ${ }^{8}$ pendientes de $0.86 \pm 0.49$ ( $\mathrm{p}=0.70)$, IHOS en pacientes de Custodia $1.89 \pm 0.78$ e Independientes $1.87 \pm .0 .74(\mathrm{p}=.93)$. IPCNT en géneros masculino $1.03 \pm 0.54$ y femenino $0.75 \pm 0.51(\mathrm{p}=.009)$. IHOS en pacientes que habitan área rural $2.41 \pm 1.25 \mathrm{y}$ área urbana $1.83 \pm 0.68(\mathrm{p}=.02)$. Relación entre la edad y la necesidad de tratamiento periodontal $(\mathrm{p}=0.001)$. Frecuencia del cepillado del grupo Independientes $2.03 \pm 0.56$ y de Custodia $2.00 \pm 0.75$ $(\mathrm{p}=.84)$. Conclusiones: No existe diferencia en el estado de salud periodontal y el grado de higiene oral entre pacientes con discapacidad Independientes y de Custodia. La mayoría de los pacientes Independientes y de Custodia tienen una necesidad de tratamiento de Instrucción de Higiene Oral (TN1) y tienen Higiene Oral Regular.

Palabras clave: enfermedad periodontal, discapacidad, higiene oral, odontopediatría

\footnotetext{
${ }^{1}$ Cirujano Maxilofacial. Coordinador del Posgrado de Periodoncia.

${ }^{2}$ Residente del Posgrado de Periodoncia

${ }^{3}$ Coordinadora del Posgrado de Odontopediatría

${ }^{4}$ Especialista en Odontopediatría y Metodología. Profesor Invitado al Posgrado de Periodoncia

${ }^{5}$ Especialista en Odontopediatría

${ }^{6}$ Especialista en Genética.

${ }^{7}$ Epidemiólogo

${ }^{8}$ Endodoncista. Adscrito al Posgrado de Periodoncia.

Lugar de realización del trabajo: Centro de Rehabilitación Infantil Teletón Altamira Tamaulipas y Universidad Autónoma de Tamaulipas. Facultad de Odontología, Posgrado de Periodoncia (México)
} 


\section{Doenças periodontais em pacientes com deficiência e sob custodia em relação a pacientes independentes}

\section{Resumo}

Objetivo: Identificar o estado de saúde periodontal em pacientes com deficiência e sob custódia versus os pacientes independentes, em sua higiene oral. Métodos: Foram examinados pacientes com deficiência, de ambos os sexos, 3 e 19 anos, que frequentam o Serviço de Odontopediatria do Centro de Reabilitação Infantil Teleton (CRIT), Tamaulipas, através de exame da cavidade bucal e aplicação do Índice de Higiene Oral Simplificado (OHI), Índice Periodontal Comunitário de Necessidade de Tratamento (IPCNT) e entrevista com o seu cuidador principal. Foram agrupados em duas categorias: Independentes e Custódia. Resultados: No total da amostra a média e o desvio padrão (DP) foi de $0,89 \pm 0,54$ IPCNT, bem como de 1,88 $\pm 0,77$ IHOS. Nos pacientes com deficiência sob custódia o IPCNT foi de 0,91 $\pm 0,57$ e Independente de $0,86 \pm 0,49(\mathrm{p}=0,70)$, OHI-S em pacientes $1,89 \pm 0,78$ e 1,87 \pm Custódia .0 .74 independente $(\mathrm{p}=.93)$. IPCNT no sexo masculino e feminino $1,03 \pm 0,540,75 \pm 0,51(\mathrm{p}=0,009)$. IHOS pacientes que vivem em áreas rurais e 2,41 $\pm 1,25 \pm$ 1,83 urbana $0,68(\mathrm{p}=.02)$. Relação entre a idade e a necessidade de tratamento periodontal ( $p$ $=0,001)$. A freqüência de escovação do grupo Independente de 2,03 $\pm 0,56$ e 2,00 $\pm 0,75$ Custódia $(\mathrm{p}=0,84)$. Conclusões: Não há diferença no estado de saúde periodontal e nível de higiene oral em pacientes com deficiência Independente e Custódia. A maioria dos pacientes independentes e sob custódia têm uma necessidade para o tratamento de Instrução de Higiene Bucal (TN1) e possuem uma higiene bucal regular.

Palavras-chave: doença periodontal, deficiência, higiene bucal, odontopediatria.

Original article

\section{Periodontal diseases in patients with disabilities in custody versus disabled patients independent}

\begin{abstract}
Objective: To identify periodontal health status in patients with disabilities of Custody versus independent patients in oral hygiene. Material and methods: We examined patients with disa-
\end{abstract}

bilities, both sexes, 3 to 19 years presenting at the Pediatric Dentistry Children's Rehabilitation Center Telethon (CRIT) Tamaulipas oral cavity by reviewing and implementing the Simplified Oral Hygiene Index (OHI) Community Periodontal Index of Treatment Need (IPCNT) and 
interview with their primary caregiver. Were grouped into two categories: Independent and Custodia. Results: In the total sample the mean and standard deviation (SD) was 0.89 IPCNT \pm 0.54 , as well as of $1.88 \pm 0.77$ IHOS. The IPCNT disabled patients was $0.91 \pm 0.57$ Custody and Independent of $0.86 \pm 0.49(\mathrm{p}=0.70)$, OHI-S in patients $1.89 \pm 0.78$ Custody and $1.87 \pm .0 .74$ Independent $(\mathrm{p}=.93)$. IPCNT in male gender and female $1.03 \pm 0.540 .75 \pm 0.51(\mathrm{p}=.009)$. IHOS patients living in rural areas and $2.41 \pm 1.25 \pm$ 1.83 urban $0.68(\mathrm{p}=.02)$. Relationship between age and the need for periodontal treatment $(\mathrm{p}=$ 0.001). Frequency of brushing the Independent group $2.03 \pm 0.56$ and $2.00 \pm$ Custody 0.75 ( $\mathrm{p}=$ .84). Conclusions: There is no difference in periodontal health status and level of oral hygiene among patients with disabilities and Independent Custody. Most Independent Custody patients have a need for treatment of Oral Hygiene Instruction (TN1) and have regular oral hygiene.

Key words: periodontal disease, disability, dental hygiene, pediatric dentistry

\section{Introducción}

La salud oral es un aspecto importante en la salud de todos los niños y es más importante para los niños con necesidades especiales de salud. Para las personas con discapacidades, el efecto de la enfermedad dental en la salud general y función es mayor que en los grupos similares sin discapacidad. Los individuos con discapacidades o enfermedades reciben menos cuidado oral que el resto de la población, a pesar del alto nivel de enfermedades dentales en ellos. ${ }^{1}$

Hay un número significativo de personas en nuestra sociedad con discapacidades y otras necesidades especiales. Muchos de estos individuos necesitan apoyo especial para recibir tratamiento dental. El número de personas con necesidades especiales que necesitan servicios de salud oral ha ido creciendo dramáticamente. ${ }^{2}$

Se estima que más de mil millones de personas viven con algún tipo de discapacidad; o sea, alrededor del $15 \%$ de la población mundial (según las estimaciones de la población mundial en 2010). Según la Encuesta Mundial de Salud, cerca de 785 millones de personas (15.6\%) de 15 años y más viven con una discapacidad, mientras que el proyecto sobre la Carga Mundial de Morbilidad estima una cifra próxima a los 975 millones (19.4\%). Sólo la Carga Mundial de Morbilidad mide las discapacidades infantiles (0-14 años), con una estimación de 95 millones de niños $(5.1 \%), 13$ millones de los cuales $(0.7 \%)$ tienen "discapacidad grave". ${ }^{3}$

Según el censo de Población y vivienda del 2010, ${ }^{4}$ en México hay 5 millones 739 mil 270 personas con algún tipo de discapacidad.

La Organización Mundial de la Salud define como una persona discapacitada "A quien por un periodo apreciable de tiempo es impedido por condiciones físicas o mentales de la completa participación en las actividades normales de su grupo de edad, incluyendo aquellas de naturaleza social, recreacional, educacional o vocacional".

Las anormalidades motoras resultantes de eventos prenatales y perinatales son numerosas y diversas en sus manifestaciones. Cuando se compromete el sistema nervioso central, el daño puede resultar en parálisis cerebral. El signo más común de la parálisis cerebral es la espasticidad o la atetosis espástica, en las que el paciente sufre movimientos involuntarios.

Los desórdenes motores observados en la parálisis cerebral distónica o más frecuentemente en 
la parálisis cerebral espástica pueden afectar el habla, la deglución y la respiración. En un estudio realizado en Brasil en pacientes con parálisis cerebral se encontró que a más severo daño neurológico, más es frecuente la presencia del reflejo de mordida y consecuentemente hay mayor riesgo de enfermedades oral en la población por la dificultad para desarrollar una adecuada higiene oral.

La mayoría de los niños con Parálisis cerebral tienen dificultades físicas en el desarrollo de actividades de la vida diaria como bañarse, vestirse, cepillarse los dientes, comer y la deambulación. Dependiendo de la severidad de la discapacidad, muchos niños con Parálisis cerebral necesitan asistencia completa o parcial para el desarrollo de estas actividades. ${ }^{7}$

Hay muchos reportes que nos muestran que las personas con discapacidades tienen más enfermedad dental, más dientes perdidos y más dificultad en la obtención de atención dental que otros miembros de la población en general.

Los resultados de series de estudios realizados en los Estados Unidos y otros países, en el estado de salud oral de jóvenes y adultos con retraso mental, discapacidades del desarrollo y una amplia gama de otras discapacidades, han enfatizado repetidamente hallazgos de pobre higiene oral, significativa incidencia de enfermedad periodontal y la resultante "mutilación de la dentición".

La prevalencia de enfermedad periodontal en pacientes con retraso mental combina los factores locales y sistémicos que influencian la susceptibilidad de la enfermedad.

En un estudio Tannenbaum y Miller reportaron que $74 \%$ de los niños institucionalizados con retraso mental de 12 a 22 años presentaban gingi- vitis crónica. Mientras que Snyder y cols. reportaron enfermedad periodontal en el $66 \%$ de los pacientes con retraso mental no institucionalizados de 1 a 19 años.

En estudios previos se ha encontrado que la enfermedad periodontal es el problema de salud oral predominante en las poblaciones con discapacidad mental y de desarrollo.

Hay una extensa documentación de un incremento en la incidencia de enfermedad periodontal y gingivitis en la población con Parálisis cerebral. La incidencia de enfermedad periodontal ha sido estimada como 3 veces más alta que la población en general. La pobre higiene oral y la hiperplasia ocasionada por la terapia con Fenitoína son los mayores contribuidores a este problema.

Sin tomar en cuenta el nivel de retraso mental en algunos pacientes con discapacidad, la higiene oral de estos individuos generalmente depende de otras personas, usualmente los padres o un guardián o los encargados en las instituciones.

Los obstáculos que impiden que estas personas logren una buena higiene oral son la falta de supervisión y asistencia, la apatía del cuidador hacia la salud dental, mayor cuidado con respecto a su discapacidad, y la falta de disponibilidad de dentistas capacitados.

Los padres de los niños con discapacidades frecuentemente evitan o ignoran los requerimientos básicos de la buena salud periodontal, y los problemas médicos y sociales hacen que la salud oral tenga una muy baja prioridad en la vida del paciente.

La dependencia y la institucionalización parecen ser factores agravantes en la enfermedad periodontal en los grupos discapacitados compara- 
dos en este estudio, las diferencias pueden ser factores del huésped más que la propia higiene por si misma. Las personas institucionalizadas y dependientes tienden a estar más severamente discapacitadas y su estado de compromiso físico puede estar asociado con una baja resistencia a los irritantes periodontales, como la placa y el cálculo.

Se ha encontrado que los niños que son más dependientes de sus cuidadores para las actividades de cuidado propio tienen altos niveles de enfermedad y mayor necesidad de tratamiento. Aquellos que requieren asistencia en el cepillado dental tienen más pobre higiene oral y más enfermedad periodontal que aquellos que pueden cepillar sus dientes por ellos mismos, lo que refleja el inadecuado cuidado oral proporcionado por los cuidadores.

La mejoría de la salud oral es una preocupación que concierne a los individuos con discapacidad, ya que la salud oral tiene tanto consecuencias locales como sistémicas. La pobre higiene oral es un factor para la co-morbilitdad cuando se asocia con enfermedad sistémica. Esto incrementa el riesgo de complicación de infecciones para pacientes que presentan enfermedad cardiaca congénita, inmunodeficiencia, diabetes, prótesis internas, así como agravando enfermedades respiratorias crónicas que son la principal causa de mortalidad en personas con discapacidad.

El desarrollo de nuevos modelos de servicios de salud oral para los individuos dependientes en un marco institucional puede dar una oportunidad de crear un nuevo paradigma de cuidado basado en la integración de servicios de salud oral con servicio social y salud general con énfasis en la prevención y la promoción de actividades de salud.

\section{Material y métodos}

El diseño del estudio fue observacional, prospectivo, descriptivo y con medición transversal de la enfermedad periodontal presente en pacientes con discapacidad de custodia o independientes. Se incluyeron pacientes de ambos sexos, de 3 a 19 años de edad que reciben terapia en el Centro de Rehabilitación Infantil Teletón, Tamaulipas, y que se atienden en el servicio de Odontopediatría y que sus tutores acepten su participación en el estudio durante el periodo de Julio a Agosto de 2011.

Se incluyeron pacientes de ambos sexos, menores de 20 años que acudan al CRIT Tamaulipas y se atiendan en el servicio de Odontopediatría y que los tutores aceptaran participar en el estudio mediante consentimiento informado y que se les pudiera realizar completo el examen clínico de cavidad oral. Excluyéndose los pacientes que no acudan acompañados de su cuidador habitual.

Las variables del estudio como independiente se consideraron independiente: discapacidad de custodia edad y género y dependiente: Índice Periodontal Comunitario para las Necesidades de Tratamiento e Índice de Higiene Oral Simplificado

Se examinaron pacientes de ambos sexos de 3 a 19 años de edad que acuden al Servicio de Odontopediatría del Centro de Rehabilitación Infantil Teletón de Tamaulipas (Tabla 1) mediante revisión de cavidad oral y aplicación del Índice de Higiene Oral Simplificado y el Índice Periodontal Comunitario de la Necesidad de Tratamiento, así como entrevista con su cuidador primario.

Para el IHOS se dividió la boca en sextantes y se revisaron seis dientes, uno de cada sextante 16 , 11, 26, 36, 31 y 46, para valorar detritos y cálculo 
tomando en cuenta una sola superficie. Las superficies dentales se examinan del borde incisal a cervical procurando revisar toda la superficie. Las superficies que se examinaron son vestibulares de 16, 11, 26 y 31, y las linguales de 36 y 46.

Los códigos utilizados para los depósitos blandos fueron 0 cuando hay ausencia de detritos o mancha extrínseca en la superficie examinada, 1 si se encuentra la presencia de detritos cubriendo no más de $1 / 3$ de la superficie del diente, o ausencia de detritos, más presencia de mancha extrínseca, 2 mediante la presencia de detritos cubriendo más de $1 / 3$ pero no más de $2 / 3$ de la superficie examinada, y 3 la presencia de detritos cubriendo más de $2 / 3$ de la superficie examinada.

Los códigos utilizados para depósitos duros fueron: 0 cuando hay ausencia de cálculo supragingival, 1 si se encuentra presencia de cálculo supragingival cubriendo no más de $1 / 3$ de la superficie examinada, 2 si se observa presencia de cálculo supragingival cubriendo más de $1 / 3$ pero no más de $2 / 3$ de la superficie examinada o bien presencia de pequeñas porciones de cálculo subgingival y 3 si hay presencia de cálculo supragingival cubriendo más de $2 / 3$ de la superficie examinada o bien una faja continúa de cálculo subgingival a lo largo de la región cervical del diente.

El promedio de detritos bucales se obtuvo sumando los valores encontrados y dividiendo entre las superficies examinadas. El mismo método se utilizó para obtener el promedio del cálculo dentario. El IHOS es la suma del promedio de detritos bucales y del cálculo dentario.

El IPCNT se llevó a cabo por medio de la sonda Hu Friedy CP-11.5B Screening Color - Coded Probe (W.H.O. probe) (Figura 6), que cuentan con una esfera en el extremo activo, lo que aumenta la sensibilidad y detección de cálculo o interferencias al momento de la medición, además de marcas $(3.5,5.5,8.5,11.5 \mathrm{~mm})$ que facilitan la clasificación del paciente.

La ausencia de cualquier signo de enfermedad tendrá un valor de 0 . La calificación 1 se registra cuando la profundidad de la bolsa es de $3 \mathrm{~mm}$ o menos pero con existencia de hemorragia al sondeo. Si el área coloreada aparece visible pero se detecta la presencia de cálculo supra o subgingival la calificación será de 2, si el área coloreada aparece parcialmente visible, la profundidad de la bolsa va de $4-5 \mathrm{~mm}$ y la calificación es de 3 . Si el área que va desde 3.5 a $5.5 \mathrm{~mm}$ desaparece en la bolsa durante la medición, la profundidad de la bolsa es de $6 \mathrm{~mm}$ o más y asigna una calificación de 4.

\section{Análisis estadístico}

Se capturaron los datos obtenidos en una base de Microsoft Excel 2010 en el que se organizaron para posteriormente realizar el análisis estadístico.

Se obtuvieron estadísticos descriptivos para las variables numéricas como IHOS, ICS, IPDBS, IPCNT, edad (Media, Desviación estándar). Estas variables numéricas se sometieron a un análisis de comprobación de normalidad de datos con la prueba Kolmogorov-Smirnov encontrando una distribución de datos asimétrica. La comparación de los grupos con los diferentes parámetros periodontales manejados en el estudio se hicieron con la prueba U-Mann WhitneyWilcoxon. Las variables cualitativas nominales analizadas con los grupos de estudio se sometieron a una prueba Chi cuadrado. Las variables ordinales como Necesidad de Tratamiento se manejaron con la prueba Kruskal-Wallis Todas 
las pruebas se manejaron a un $\alpha=0.05$ en el programa estadístico PASW 18.0 (SPSS).

\section{Resultados}

En el presente estudio se tomó una muestra de 104 pacientes con discapacidad (53 hombres y 51 mujeres), de origen rural 10 pacientes $(9.61 \%)$ y urbano 94 pacientes $(90.39 \%)$, con edades entre los 3 y 19 años que reciben tratamiento en el Centro de Rehabilitación Infantil Teletón Tamaulipas en el Servicio de Odontopediatría, a los cuales agrupamos en 2 categorías: Independientes (36) y de Custodia (68) en la realización de su higiene oral.

Analizados los datos se da a conocer la distribución de grupos (Independiente y de Custodia) por género de la muestra total de pacientes con discapacidad, edad y lugar de residencia.

El IHOS en los pacientes que viven en áreas rurales (10) es de $2.41 \pm 1.25$ y el de los que viven en áreas urbanas (94) es de $1.83 \pm 0.68$ ( $\mathrm{p}=0.02)$. El IPCNT en el grupo de Independientes fue de $0.86 \pm 0.49$ y el del grupo de Custodia 0.91 ( $p=.70)$. Mientras que el resultado del IPCNT por género mostró ser significativamente mayor en pacientes del género masculino $1.03 \pm 0.54$, mientras que en el género femenino fue de $0.75 \pm 0.51$ ( $\mathrm{p}=.009)$.

Se observan además los resultados del IPDB-S, IC-S e IHOS encontrados en la totalidad de la muestra. Aparecen los resultados del IPDB-S, IC$S$, IHOS e IPCNT por grupos Independiente y De custodia. El IPDB-S nos muestra valores similares para ambos grupos que califican como una condición regular $(\mathrm{p}<0.94)$, el índice de cálculo nos muestran valores de una condición buena en ambos grupos de estudio( $p<0.65)$, mientras que el IHOS define una condición general de regular higiene oral en ambos grupos $(\mathrm{p}<0.91)$. El IPCNT resulta en una necesidad de tratamiento 1 $(\mathrm{p}<0.94)$ interpretándose Mejorar higiene bucal.
Tabla 1. Distribución por género y edad en cada grupo (Independientes y de custodia) de la muestra de pacientes con discapacidad y lugar de residencia del total de la muestra.

\begin{tabular}{|c|c|c|}
\hline Grupo & Independiente & De custodia \\
\hline Índices orales & Media DE & Media DE \\
\hline $\begin{array}{l}\text { Edad del } \\
\text { Paciente }\end{array}$ & $11.36 \pm 3.7$ & $9.04 \pm 4.7$ \\
\hline IPDB-S & $1.66 \pm 0.59$ & $1.65 \pm 0.53$ \\
\hline IC-S & $0.21 \pm 0.38$ & $0.24 \pm 0.49$ \\
\hline IHOS & $1.87 \pm 0.74$ & $1.89 \pm 0.78$ \\
\hline IPCNT & $0.86 \pm 0.49$ & $0.91 \pm 0.57$ \\
\hline $\begin{array}{l}\text { Género del } \\
\text { paciente }\end{array}$ & No. $(\%)$ & No. $(\%)$ \\
\hline Masculino & $20(55.6 \%)$ & $33(28.5 \%)$ \\
\hline Femenino & $16(44.4 \%)$ & $35(51.5 \%)$ \\
\hline \multicolumn{3}{|l|}{ Necesidad Tto. } \\
\hline NT 0 & $8(22.2 \%)$ & $21(30.9 \%)$ \\
\hline NT 1 & $24(66.7 \%)$ & $39(57.4 \%)$ \\
\hline NT 2 & $4(11.1 \%)$ & $8(11.8 \%)$ \\
\hline NT 3 & $0(0 \%)$ & $1(1.5 \%)$ \\
\hline NT 4 & $0(0 \%)$ & $0(0 \%)$ \\
\hline \multicolumn{3}{|l|}{$\begin{array}{l}\text { Parentesco del } \\
\text { Cuidador }\end{array}$} \\
\hline Padre & $3(8.3 \%)$ & $5(7.4 \%)$ \\
\hline Madre & $30(83.3 \%)$ & $59(86.8 \%)$ \\
\hline Abuelo & $1(2.8 \%)$ & $3(4.4 \%)$ \\
\hline Tío & $1(2.8 \%)$ & $1(1.5 \%)$ \\
\hline Otro & $1(2.8 \%)$ & $0(0 \%)$ \\
\hline \multicolumn{3}{|l|}{$\begin{array}{l}\text { Edad del } \\
\text { Cuidador }\end{array}$} \\
\hline 15-25 años & $1(2.8 \%)$ & $10(14.7 \%)$ \\
\hline 26-35 años & $8(22.3 \%)$ & $24(35.3 \%)$ \\
\hline 36-45 años & $16(44.4 \%)$ & $25(36.8 \%)$ \\
\hline 46-55 años & $11(30.6 \%)$ & $7(10.3 \%)$ \\
\hline 56-66 años & $0(0 \%)$ & $2(2.9 \%)$ \\
\hline
\end{tabular}


Se encontraron las siguientes diferencias por grado de dependencia y necesidad de tratamiento mediante la prueba de Chi cuadrada NT $0(\mathrm{p}<0.24)$, NT $1(p<0.23)$, NT $2(p<0.59)$, NT $3(p<0.1)$

Se describe el parentesco del cuidador primario entre los dos grupos de la muestra estudiada (Independientes y de Custodia). En la Tabla 10 se enumeran frecuencias y porcentajes de los grupos de edades del cuidador primario de los pacientes Independientes y de custodia de la muestra de pacientes con discapacidad.

Asímismo se detallan las frecuencias y porcentajes de Necesidad de tratamiento en cada grupo estudiado $(p=0.48)$.

Las frecuencias y porcentajes de los resultados del índice de Higiene Oral Simplificado entre los diferentes grupos de diagnóstico de pacientes con discapacidad se detallan en la tabla 2.

Tabla 2. Resultados Índice de Higiene Oral Simplificado por grupos de diagnóstico de la muestra total de pacientes con discapacidad. $p<0.93$

\begin{tabular}{|c|c|c|c|c|c|}
\hline Grupos de edad & No. & Buena & Regular & Mala & Total \\
\hline $3-6$ & 34 & $43.9 \%$ & $32.5 \%$ & $18.2 \%$ & $32.7 \%$ \\
\hline $7-9$ & 15 & $6.3 \%$ & $18.2 \%$ & $0 \%$ & $14.4 \%$ \\
\hline $10-12$ & 24 & $25.1 \%$ & $22.1 \%$ & $27.3 \%$ & $23.1 \%$ \\
\hline $13-15$ & 16 & $12.5 \%$ & $14.3 \%$ & $27.3 \%$ & $15.4 \%$ \\
\hline $16-19$ & 15 & $12.5 \%$ & $13.0 \%$ & $27.3 \%$ & $14.4 \%$ \\
\hline
\end{tabular}

Tabla 3. Frecuencias y porcentajes de pacientes en los diferentes grupos de diagnóstico de pacientes con discapacidad. $p<0.61$

\begin{tabular}{|c|c|c|c|c|c|}
\hline Grupos Dx. & MMCE & ENNM & EOAR & LOPC & EGOC \\
\hline Higiene oral & & & & & \\
\hline Buena & $2(28.6 \%)$ & $3(20.0 \%)$ & $1(11.1 \%)$ & $9(15.3 \%)$ & $1(7.1 \%)$ \\
\hline Regular & $5(71.4 \%)$ & $11(73.3 \%)$ & $7(77.8 \%)$ & $43(72.9 \%)$ & $11(78.6 \%)$ \\
\hline Mala & $0(0 \%)$ & $1(6.7 \%)$ & $1(11.1 \%)$ & $7(11.9 \%)$ & $2(14.3 \%)$ \\
\hline Necesidad Tto. & & & & & \\
\hline NT 0 & $4(51.1)$ & $4(26.7 \%)$ & $3(33.3 \%)$ & $15(25.4 \%)$ & $3(21.4 \%)$ \\
\hline NT 1 & $2(28.6 \%)$ & $8(53.3 \%)$ & $6(66.7 \%)$ & $37(62.7 \%)$ & $10(71.4 \%)$ \\
\hline NT 2 & $1(14.3 \%)$ & $3(20.0 \%)$ & $0(0 \%)$ & $6(10.2 \%)$ & $1(7.1 \%)$ \\
\hline NT 3 & $0(0 \%)$ & $0(0 \%)$ & $0(0 \%)$ & $1(1.7 \%)$ & $0(0 \%)$ \\
\hline NT 4 & $0(0 \%)$ & $0(0 \%)$ & $0(0 \%)$ & $0(0 \%)$ & $0(0 \%)$ \\
\hline
\end{tabular}

$M M C E=$ Mielomeningocele,$E N N M=$ Enf. Neuromusculares, EOAR $=$ Enf. Osteoarticulares, $L O P C=$ Lesión o Parálisis Cerebral, EGOC = Enf. Genéticas o Congénitas. 
Tabla 4. Necesidad de tratamiento periodontal por grupos de edad de la muestra total de pacientes con discapacidad. $p$ $<0.001$.

\begin{tabular}{|c|c|c|c|c|c|}
\hline Grupos de edad & NT 0 & NT 1 & NT 2 & NT 3 & NT 4 \\
\hline $3-6$ & $10(34.5 \%)$ & $24(38 \%)$ & $0(0 \%)$ & $0(0 \%)$ & $0(0 \%)$ \\
\hline $7-9$ & $7(24.1 \%)$ & $8(12.7 \%)$ & $0(0 \%)$ & $0(0 \%)$ & $0(0 \%)$ \\
\hline $10-12$ & $8(27.5 \%)$ & $15(23.8 \%)$ & $1(9.1 \%)$ & $0(0 \%)$ & $0(0 \%)$ \\
\hline $13-15$ & $2(6.9 \%)$ & $10(15.9 \%)$ & $4(36.4 \%)$ & $0(0 \%)$ & $0(0 \%)$ \\
\hline $16-19$ & $2(6.8) \%$ & $6(9.6 \%)$ & $6(54.6 \%)$ & $1(100 \%)$ & $0(0 \%)$ \\
\hline TOTAL & $29(27.9 \%)$ & $63(60.6 \%)$ & $11(10.6 \%)$ & $1(1.0 \%)$ & $0(0 \%)$ \\
\hline
\end{tabular}

Tabla 5. Índice de Higiene Oral Simplificado de los pacientes con discapacidad según la escolaridad de su cuidador primario. $p<0.02$.

\begin{tabular}{|c|c|c|c|c|}
\hline $\begin{array}{c}\text { IHOS del paciente } \\
\text { Escolaridad CP }\end{array}$ & Buena & Regular & Mala & Total \\
\hline Sin estudios & $0(0 \%)$ & $0(0 \%)$ & $2(100 \%)$ & $2(1.9 \%)$ \\
\hline Primaria & $4(23.5 \%)$ & $11(64.7 \%)$ & $2(11.8 \%)$ & $17(16.3 \%)$ \\
\hline Secundaria & $5(15.2 \%)$ & $27(81.8 \%)$ & $1(3.9 \%)$ & $33(31.7 \%)$ \\
\hline Bachillerato & $4(16.7 \%)$ & $16(66.7 \%)$ & $4(16.7 \%)$ & $24(23.1 \%)$ \\
\hline Carrera Técnica & $0(0 \%)$ & $4(100 \%)$ & $0(0 \%)$ & $4(3.8 \%)$ \\
\hline Profesional & $2(11.8 \%)$ & $13(76.5 \%)$ & $2(11.8 \%)$ & $17(16.3 \%)$ \\
\hline Posgrado & $1(14.3 \%)$ & $6(85.7 \%)$ & $0(0 \%)$ & $7(6.7 \%)$ \\
\hline TOTAL & $16(15.4 \%)$ & $77(74.0 \%)$ & $11(10.6 \%)$ & $104(100 \%)$ \\
\hline
\end{tabular}

En la tabla 4 se describen las frecuencias y porcentajes de la Necesidad de Tratamiento Periodontal por grupos de edad en la totalidad de la muestra de pacientes con discapacidad. Se encontró una relación, observando que a mayor edad mayor Necesidad de Tratamiento Periodontal $(\mathrm{p}=.001)$.
Dentro de la Tabla 5 se observan resultados significativos $(\mathrm{p}=.02)$ con respecto al Índice de Higiene Oral Simplificado y el nivel de escolaridad del cuidador primario.

En la tabla 6 se describe en frecuencias y porcentajes la Necesidad de Tratamiento Periodon- 
Tabla 6. Necesidad de tratamiento periodontal de pacientes con discapacidad según el grado de escolaridad del cuidador primario. $p<0.55$.

\begin{tabular}{|c|c|c|c|c|c|c|}
\hline $\begin{array}{c}\text { NT del } \\
\text { paciente } \\
\text { Escolaridad } \\
\text { CP }\end{array}$ & NT 0 & NT 1 & NT 2 & NT 3 & NT4 & TOTAL \\
\hline Sin estudios & $0(0 \%)$ & $1(50 \%)$ & $1(50 \%)$ & $0(0 \%)$ & $0(0 \%)$ & $2(1.9 \%)$ \\
\hline Primaria & $5(29.4 \%)$ & $8(47.1 \%)$ & $3(17.6 \%)$ & $1(5.9 \%)$ & $0(0 \%)$ & $17(16.3 \%)$ \\
\hline Secundaria & $8(24.2 \%)$ & $23(69.7 \%)$ & $2(6.1 \%)$ & $0(0 \%)$ & $0(0 \%)$ & $33(31.7 \%)$ \\
\hline Bachillerato & $8(33.3 \%)$ & $14(58.3 \%)$ & $2(8.3 \%)$ & $0(0 \%)$ & $0(0 \%)$ & $24(23.1 \%)$ \\
\hline Carrera Téc- & $1(25.0 \%)$ & $3(75.0 \%)$ & $0(0 \%)$ & $0(0 \%)$ & $0(0 \%)$ & $4(3.8 \%)$ \\
\hline nica & $7(41.2 \%)$ & $8(47.1 \%)$ & $2(11.8 \%)$ & $0(0 \%)$ & $0(0 \%)$ & $17(16.3 \%)$ \\
\hline Profesional & $0(0 \%)$ & $6(85.7 \%)$ & $1(14.3 \%)$ & $0(0 \%)$ & $0(0 \%)$ & $7(6.7 \%)$ \\
\hline Posgrado & $29(27.9)$ & $63(60.6 \%)$ & $11(10.6 \%)$ & $1(1.0 \%)$ & $0(0 \%)$ & $104(100 \%)$ \\
\hline TOTAL & & & &
\end{tabular}

tal de los pacientes de la totalidad de la muestra con discapacidades según la escolaridad del cuidador primario.

\section{Discusión}

Las enfermedades periodontales se encuentran entre los padecimientos que más frecuentemente afectan a niños y adolescentes, y se ha encontrado que los pacientes con algún grado de discapacidad pueden ser más susceptibles a las afectaciones periodontales. Se ha sugerido que esta situación se debe a la falta de una adecuada higiene oral dadas las condiciones, físicas, motoras e intelectuales que sufren estos individuos.

Debido a las limitaciones en la cooperación de los pacientes en el estudio, la examinación pe- riodontal meticulosa fue difícil, sin embargo se registraron cuidadosamente el sangrado, la presencia o ausencia de cálculo y placa dentobacteriana, así como la profundidad al sondeo con el objetivo de conseguir la mayor exactitud en los resultados de estas variables.

Después de analizar los resultados no encontramos diferencias estadísticamente significativas entre el grado de dependencia en cuanto a los valores de los índices periodontales. Creemos que el hecho de que nuestra muestra forma parte del Centro de Rehabilitación Infantil Teletón, que es una institución que está atenta de las necesidades de los pacientes y que no sólo abarca la rehabilitación física de una discapacidad, sino que también incluye la salud oral, pudo haber sido la causa de que esta situación se presenta- 
ra. Los pacientes que se incluyeron en el estudio reciben atención en el servicio de Odontopediatría, por lo que son constantemente monitoreados y reciben instrucciones de higiene oral. No encontramos enfermedad periodontal grave en ninguno de los casos, y la higiene oral en general fue regular.

Nuestros resultados no coinciden con los reportados por la mayoría de las investigaciones previas $^{13,18,19}$, que han encontrado peor estado de salud periodontal e higiene en aquellos pacientes que son más dependientes de sus cuidadores o que tienen mayor grado de retraso mental ${ }^{27}$ o daño neurológico ${ }^{5}$. En nuestro estudio se observó similar estado de salud periodontal entre pacientes Independientes y de custodia, esto puede deberse a que en los estudios previos se ha tomado la muestra en escuelas de educación especial y no en centros de rehabilitación infantil en el que los pacientes reciben atención odontológica periódica como lo fue en nuestro caso.

Además es conocido que para mantener una adecuada higiene y salud oral no solo se requiere tener la capacidad física para realizar el cepillado dental y utilizar los aditamentos de higiene oral, sino que es necesario tener la disciplina y el hábito de realizar el cepillado dental. En muchas ocasiones esta disciplina no está presente en los pacientes con discapacidad debido a la sobreprotección de los padres o a la falta de importancia que ellos mismos le dan al cuidado oral. Con ello se enfatiza la importancia que representa el que un paciente con discapacidad reciba atención en un centro de rehabilitación que considere todos los aspectos relacionados a la salud.

La necesidad de tratamiento más prevalente fue la Instrucción de Higiene Oral (TN1), esta fue seguida por los pacientes que no requerían tra- tamiento (TN0) y posteriormente la Instrucción de Higiene Oral más Raspado y Alisado radicular y colocación de restauraciones en caso necesario (TN2). La necesidad de tratamiento ${ }^{3}$ solo se observó en el 1\% de la muestra y no se encontró TN4 en ningún sujeto del estudio. Estos valores pueden relacionarse con la edad de los pacientes, ya que se ha encontrado que los problemas periodontales aumentan con la edad ${ }^{10,11,12,24,27,28}$. Al respecto en nuestra investigación observamos una relación que señala que a mayor edad mayor necesidad de tratamiento periodontal.

Se revisaron trabajos realizados en poblaciones sin discapacidad en México, Hernández $\mathrm{P}$ y cols. ${ }^{24}$ encontraron en pacientes escolares de Yucatán una media de IHOS de 1.43. En nuestro estudio este resultado alcanzó 1.88. Ambos valores entran dentro del rango de una higiene bucal regular. Mientras que en Sinaloa VillalobosRodelo y cols. ${ }^{25}$ encontraron una media de valor de IHOS de 1.10 que representa una buena higiene bucal. Los estudios anteriormente citados evaluaron una población sin discapacidad, si los comparamos con nuestra muestra de pacientes con discapacidad, podemos observar que los valores no son tan diferentes. Por esto, enfatizamos el hecho de que nuestra muestra es parte de un centro de rehabilitación infantil donde la atención odontológica forma un componente importante en la salud. Esto refleja la atención cuidadosa que el CRIT ofrece a todas las necesidades que sus pacientes tienen, además de la capacitación y concientización que los colaboradores de este centro les brindan a los padres y cuidadores primarios sobre la importancia de la salud oral en el estado general del niño.

En nuestros resultados encontramos que los pacientes del sexo masculino tienen más altos valores de IPCNT. Este hallazgo se observó de manera similar reportado de Kumar y cols. ${ }^{28}$ donde 
se determinó que los pacientes con discapacidad de sexo masculino tienen un peor grado de higiene oral y un peor estado periodontal.

Encontramos que aquellos pacientes que viven en áreas rurales tienen más altos valores en el IHOS, esto coincide con algunos autores como Hernández P y cols..$^{24}$ quienes afirman que la enfermedad periodontal en la población rural es mayor que en la urbana. Villalobos-Rodelo y cols. ${ }^{25}$ reportaron que los niños con mejor estado socioeconómico tienen mejor higiene oral. Así mismo, Glassman y cols. ${ }^{16}$ afirman que hay una relación entre el estado socioeconómico y el grado de salud oral.

En nuestro estudio solo identificamos a 2 cuidadores primarios sin estudios en los que observamos que los niños dependientes de ellos presentaron una mala higiene oral, observamos una tendencia de higiene oral regular en todos los grados de escolaridad, con excepción del anteriormente señalado. En relación a esto, autores como Kumar y cols. $^{28}$ determinaron que el nivel de educación de los padres, así como el nivel socioeconómico tienen influencia sobre la higiene oral y el estado periodontal de los pacientes con discapacidad mental. Es importante subrayar esto, ya que Subasi y cols. ${ }^{6}$ también destacan en su estudio el importante papel que juegan los padres en la formación de los hábitos de higiene oral de los pacientes y la relación de los pobres hábitos de cuidado dental con el nivel de educación de las madres.

La media de la frecuencia diaria del cepillado fue de 2 veces al día para ambos grupos de estudio, este dato fue obtenido por la entrevista con el cuidador primario del paciente, y no siempre se observó relación entre la cantidad de frecuencias de cepillado con el IHOS e IPCNT, por lo que este dato puede ser solo producto de los deseos de los cuidadores por aparentar tener un mejor cuidado en la salud oral del paciente con discapacidad.

La frecuencia diaria de cepillado es un aspecto muy importante en el cuidado de la salud e higiene oral, ya que cuando existe un hábito de cepillado dental se puede mejorar la técnica del mismo. En el caso de nuestra muestra, los pacientes de Custodia dependen para su higiene de sus cuidadores primarios, quienes probablemente pueden tener hábitos irregulares de higiene, ya que es bien conocida la elevada prevalencia de enfermedad periodontal en la población mexicana. ${ }^{31}$ Por lo que creemos que si un cuidador primario presenta algún tipo de enfermedad periodontal, como es presumible observar dada la prevalencia de esta enfermedad, es difícil suponer que este cuidador incida favorablemente en la salud oral de alguna persona con discapacidad.

Es deseable la realización de futuras investigaciones que encaminen programas de prevención en este tipo de población, dada la vulnerabilidad que presentan por su condición de discapacidad.

La visión de apertura del CRIT en la colaboración con otras instituciones de salud o educativas superiores especializadas permiten establecer parámetros de evaluación con respecto a la salud de la población que acude y que reciban los beneficios que tienen como objetivo proporcionar una óptima calidad de vida.

\section{Conclusiones}

Bajo las condiciones y limitaciones en que se llevó nuestra investigación, se destacan las siguientes conclusiones:

- No existe diferencia en el estado de salud periodontal y el grado de higiene oral entre los pacientes con discapacidad Independientes y de Custodia. 
- La mayoría de los pacientes con Discapacidad Independientes y de Custodia tienen una necesidad de tratamiento de Instrucción de Higiene Oral (TN1).

- Los pacientes con Discapacidad Independientes y de Custodia presentaron Higiene Oral Regular.
- La necesidad de tratamiento periodontal aumenta con la edad en pacientes con discapacidad tanto de Custodia como Independientes.

- El IHOS en pacientes con discapacidad que viven en áreas rurales es mayor a la observada en los pacientes que viven en áreas urbanas.

\section{Referencias}

1. Bhambal A, Jain M, Saxena S, Kothari S. Oral health preventive protocol for mentally disabled subjects- A review. J Adv Dental Research. 2011; 2(1): 21-26.

2. Glassman P. A review of guidelines for sedation, anesthesia, and alternative interventions for people with special needs. Spec Care Dentist. 2009; 29(1): 9-16.

3. Organización Mundial de la Salud. Informe mundial sobre la discapacidad. 2011. (http: / / www.who.int/ disabilities / world_report/2011/summary_es.pdf)

4. INEGI. Censo de Población y vivienda 2010. México 2010.

5. Kaufman E, Meyer S, Wolnerman J, Gilai A. Transient suppression of involuntary movements in cerebral palsy patients during dental treatment. Anesth Prog. 1991; 38 (6): 200-205.

6. Dos Santos M, Nogueira M. Infantile reflexes and their effects on dental caries and oral hygiene in cerebral palsy individuals. Journal of Oral Rehabilitation. 2005; 32: 880-885.

7. Subasi F, Mumcu G, Koksal L, Cimilli H, Bitlis D. Factors affecting oral health habits among children with cerebral palsy: Pilot study. Pediatrics International. 2007; 49: 853-857.

8. Naciones Unidas Derechos Humanos. Oficina del Alto Comisionado de las Naciones Unidas para los Derechos Humanos. Convención sobre los derechos de los niños. 1990. (http: / / www2.ohchr.org/spanish/law/crc.htm)

9. Waldman H, Perlman S, Swerdloff M. Periodontics and Patients With Special Needs. J Periodontology. 2000; Vol. 71(2): 330-333.

10. Leary BA, Zucker SB. Teaching preventive dentistry to adolescents with cerebral palsy. Spec Care Dentist. 1981; Vol. 1 No. 1. January-February: 13-17.

11. Stiefel D. Dental Care Considerations for Disabled Adults. Spec Care Dentist. 2002; 22(3): 26S-39S.

12. Armitage GC. Development of a Clasification System for Periodontal Disease and Conditions. Ann Periodontol. 1999; 4:1-6

13. Carranza, Newmann, Takei, Klollevold,. Periodontología Clínica. 10a edición. China. Ed. Mc Graw Hill; 2010. p. 100109.

14. Mariotti A. Dental Plaque Induced gingival diseases. Ann Periodontol. 1999; 4:7-17

15. Caton JG, Williams R, Zappa U, Claffey N, Greenwell H, Mahanonda R, Mariotti,Zackin J, Consensus report: Dental Plaque-Induced Gingival diseases. Ann Periodontol. 1999; 4:18-20.

16. Oh TJ, Eber R, Wang HL. Periodontal diseases in the child and adolescent. J Clinical Periodontol. 2002; 29: 400-410.

17. Bimstein E, Sapir S, Houri-Haddad Y, Dibart S, Van Dyke TE, Shapira L. The Relationship Between Porphyromonas gingivalis Infection and Local and Systemic Factors in Children. J Periodontol. 2004;75: 1371-1376. 
Fermín-Guerrero-Del Ángel, Lilia Alejandra-Dávila-Salazar, Hilda-Issasi-Hernández, Enrique E -Huitzil -Muñoz, Ariel-Cabrera-Salazar, Marcela-Fragoso- Benítez, Rogelio-Oliver-Parra, José Martín-Torres-Benítez

18. Tesini D. An annotated review of the literature of dental caries and periodontal disease in mentally retarded individuals. Spec Care Dentist. 1981; Mar-Apr;1(2): 75-87.

19. Hernández J, Tello T, Hernández F, Rosette M. Enfermedad periodontal: prevalencia y algunos factores asociados en escolares de una región mexicana. Revista ADM. 2000; Vol. LVII. No. 6. Nov- Dic: 222-230.

20. Villalobos-Rodelo J, Medina-Solis C, Maupomé G, Vallejos-Sánchez A, Lau-Rojo L, Ponce de León-Viedas M. Socioeconomic and sociodemografic variables associated with oral hygiene status in mexican schoolchildren aged 6 to 12 years. J Periodontol. 2007; Vol. 78. Number 5. May: 816-822.

21. Stiefel DJ, Truelove EL, Mandel LS. A comparison of oral health in spinal cord injury and other disability groups. Spec Care in Dentist. 1993; Vol.13 No. 6: 229-235.

22. Southern association of institutional dentists. Cerebral palsy: A review for dental professionals. Self-study course. Module 4. (http: / / saiddent.org/modules/12_module4.pdf)

23. Idaira Y, Nomura Y, Tamaki Y, Katsumura S, Kodama S, Kurata K, Asafa Y. Factors affecting the oral condition of patients with severe motor and intellectual disabilities. Oral Dis. 2008; 14: 435-424

24. Naka S, Yamana A, Nakano K, Okawa R, Fujita K, Kojima A, Nemoto H, Nomura R, Matsumoto M, Ooshima T. Distribution of periodontopathic bacterial species in Japanese children with developmental disabilities. BMC Oral health 2009; 9:24. (http: / / www.biomedcentral.com/1472-6831/9/24)

25. Shao Feng H, Mendes Pinheiro IC, Rosa Grande S, Mendes Pannuti C, Nunes Barros FJ, Moreira Lotufo RF. Effectiveness of a triclosanhopolymerdentifrice on dental plaque and gingivitis in Brazilian individuals with cerebral palsy. Spec Care Dentist. 2007; 27(4): 144-148.

26. Kahabuka FK, Ndalahwa M. Parental oral health care of mentally retarded children in Ilala municipality, Dar es Salaam, Tanzania. Int J Dent Hygiene. 2006; 4: 145-149.

27. Nematollahi H, Makarem A, Noghani AR. Periodontal Treatment Needs Amongst 9-14 Year-old Institutionalized Mentally Retarded Children in Mashhad, IRAN. Shiraz Univ Dent J 2010; Vol.10, Supplement: 15-20.

28. Kumar S, Sharma J, Duraiswamy P, Kulkami S. Determinants for oral hygiene and periodontal status among mentally disabled children and adolescents. J Indian Soc of Pedod Prevent Dent. Jul- Sept 2009. Issue 3, Vol. 27: $151-157$.

29. Figueiredo LC, Feres M, Salvador SL. Halitosis and periodontal disease in subjects with mental disabilities. Oral Dis. 2005; 11: 83-85.

30. Sakellari D, Arapostathis F, Konstantinides A. Periodontal conditions and subgingival microflora in Down syndrome patients. J Clinical Periodontol. 2005; 32: 684-690.

31. Glassman P., Subar P. Creating and maintaining oral health for dependent people in institutional settings. J Public Health Dent. 2010; 70: S40- S48

32. Desai M, Brearley Messer L, Calache H. A study of the dental treatment needs of children with disabilities in Melbourne, Australia. Aust Dent J. 2001; 46(1): 41-50

33. Thornton J, Al- Zahid S, Campbell V, Marchetti A, Bradley E. Oral hygiene levels and periodontal disease prevalence among residents with mental retardation at various residential settings. Spec Care Dentist. 1989 Nov-Dec: 186- 190 .

34. Hennequin M, Moysan V, Jourdan D, Dorin M, Nicolas E. Inequalities in oral health for children with disabilities: a French national survey in special schools. PLoS ONE. 2008; 3 (6): e2564: 1- 11.

35. Smith G, Rooney Y, Nunn J. Provision of dental care for special care patients: the view of Irish dentists in the Republic of Ireland. J Ir Dent Assoc. 2009; 56 (2): 80- 84.

36. Siqueira W, Rodrigues Santos M, Elagovan S, Simoes A, Nicolau J. The influence of valproic acid on salivary pH in children with cerebral palsy. Special Care Dentist. 2007; 27(2): 64-6. 
37. Medina-Solis CE, Maupomé G, Avila-Burgos L, Pérez-Núñez R, Pelcastre-Villafuerte B, Pontigo-Loyola A. Políticas de salud bucal en México: Disminuir las principales enfermedades. Una descripción. Rev Biomed 2006; 17: 269-286.

38. Castro Bernal C, Fernández Pratts M, Carrasco Gutiérrez R. Índices Epidemiológicos Orales. México. Facultad de Estomatología BUAP; 2009. p. 105- 118.

Texto Traducido por: Paulo César B. Rédua

Recibido: 06 -03-2012

Aceptado: 19-03-2012

Correspondencia:ferguean@gmail.com 